\title{
A new experimental phase diagram investigation of $\mathrm{Cu}-\mathrm{Sb}$
}

\author{
Siegfried Fürtauer $\cdot$ Hans Flandorfer
}

Received: 16 January 2012/ Accepted: 9 February 2012

(C) The Author(s) 2012. This article is published with open access at Springerlink.com

\begin{abstract}
The binary system $\mathrm{Cu}-\mathrm{Sb}$ is a constituent system that is studied in investigations of technically important ternary and quaternary alloy systems (e.g., casting alloys and lead-free solders). Although this binary system has been thoroughly investigated over the last century, there are still some uncertainties regarding its high-temperature phases. Thus, parts of its phase diagram have been drawn with dashed lines in reviews published in the literature. The aim of this work was to resolve these uncertainties in the current phase diagram of $\mathrm{Cu}-\mathrm{Sb}$ by performing XRD, SEM-EDX, EPMA, and DTA. The results from thermal analysis agreed well with those given in the literature, although some modifications due to the invariant reaction temperatures were necessary. In particular, reactions located on the $\mathrm{Cu}$-rich side of the nonquenchable high-temperature $\beta$ phase $\left(\mathrm{BiF}_{3}\right.$-type) left considerable scope for interpretation. Generally, the structural descriptions of the various binary phases given in the literature were verified. The range of homogeneity of the $\varepsilon$ phase $\left(\mathrm{Cu}_{3} \mathrm{Ti}\right.$ type) was found to be higher on the Sb-rich side. Most of the reaction temperatures were verified, but a few had to be revised, such as the eutectoid reaction $\beta \rightarrow \varepsilon+\eta$ at $440{ }^{\circ} \mathrm{C}$ (found to occur at $427{ }^{\circ} \mathrm{C}$ in this work) and the eutectoid reaction $\gamma \rightarrow(\mathrm{Cu})+\delta$ at $400{ }^{\circ} \mathrm{C}$ (found to occur at $440{ }^{\circ} \mathrm{C}$ in this work). Further phase transformations that had previously only been estimated were confirmed, and their characteristic temperatures were determined.
\end{abstract}

Dedicated to O. Prof. Dr. Herbert Ipser on the occasion of his 65 th birthday.

S. Fürtauer $\cdot$ H. Flandorfer $(\square)$

Department of Inorganic Chemistry/Materials Chemistry,

University of Vienna, Währingerstraße 42, 1090 Vienna, Austria

e-mail: hans.flandorfer@univie.ac.at
Keywords Phase diagrams - Phase transitions . $\mathrm{X}$-ray structure determination · Thermodynamics

\section{Introduction}

\section{Lead-free soldering}

Compared to, say, lead-acid accumulators, solders used in electronics utilize only a relatively small proportion of the lead consumed worldwide. However, recycling lead from electronic waste is a complicated task, and it pollutes the environment when deposited in landfills and incinerator plants. In the European Union, the use of lead-containing solders has been prohibited since 2006, although there are unfortunately many exceptions for special applications. The electronics industry has therefore tried to phase-in the use of solders containing other, less harmful, materials than lead over the last decade. While the development of lead-free lowtemperature soft solders (melting point $\sim 180-230{ }^{\circ} \mathrm{C}$ ) is fairly advanced, research into lead-free high-temperature soft solders (melting range $>230-350{ }^{\circ} \mathrm{C}$ ) is still in progress. In order to perform a systematic search for appropriate alloy systems, some fundamental data on phase relations and thermochemical properties are essential. COST Action MP0602 will lead to the creation of an encyclopedic database containing data on several different binary and ternary alloy systems. Alloy systems containing the components of leadfree solder and substrate materials are of particular interest for inclusion in this database. The $\mathrm{Cu}-\mathrm{Sb}$ system is a possible binary constituent of lead-free solder systems. Indeed, $\mathrm{Sb}$ is a component of some lead-free solders that are already available on the market (e.g., $\mathrm{Ag}-\mathrm{Sb}-\mathrm{Sn}$ or $\mathrm{Cu}-\mathrm{Sb}-\mathrm{Sn}$ ), and copper is the most commonly used substrate, as well as a potential component of the solder itself. 
Despite the fact that there is already a considerable amount of data on the $\mathrm{Cu}-\mathrm{Sb}$ system, some ambiguities were noticed when a literature search focusing on this system was performed. This primarily affects the hightemperature phase ( $\beta$ phase, $\mathrm{BiF}_{3}$ type), which cannot be stabilized at room temperature by quenching. Thus, the aim of the work described in the present paper was to improve the current version of the phase diagram for the $\mathrm{Cu}-\mathrm{Sb}$ system by incorporating data gained from new experiments and by critically assessing the available data in the relevant literature. This work will therefore contribute valuable information to the lead-free solder database and lead to better thermodynamic descriptions of this binary system (see $[1,2])$ and derived higher-order systems via the CALPHAD approach.

\section{Literature review}

The $\mathrm{Cu}-\mathrm{Sb}$ phase diagram, as drawn in Massalski [3], is presented in Fig. 1. Invariant reactions are listed in Table 1 and crystallographic data in Table 2, which were taken from works by several authors (see [4-11]).

The $\alpha$ phase is $\mathrm{Cu}$ containing $\mathrm{Sb}$ with extended solubility. The maximum solubility of Sb occurs at 5.8 at $\% \mathrm{Sb}$ and $645{ }^{\circ} \mathrm{C}$. In contrast to this, there is nearly no solubility of $\mathrm{Cu}$ in $\mathrm{Sb}$. The $\beta$ phase, which is a high-temperature phase, melts congruently at $683{ }^{\circ} \mathrm{C}$. It crystallizes in a cubic $\mathrm{BiF}_{3}$-type structure $\left(\mathrm{DO}_{3}\right)$ with the space group $\mathrm{Fm}$ $3 m$. At the liquid melt, the Sb-rich $\beta$ forms the $\eta$ phase in a peritectic reaction $\left(586{ }^{\circ} \mathrm{C}\right)$. On the $\mathrm{Cu}$-rich side, $\beta$ and $(\mathrm{Cu})$ are formed eutectically at $645^{\circ} \mathrm{C}$. The $\beta$ phase decomposes in a eutectoid reaction at $440{ }^{\circ} \mathrm{C}$ into $\varepsilon$ and $\eta$. Schubert and Illschner first published this reaction [12], and Heumann and Heinemann [13] subsequently proposed the eutectoid reaction $\beta \rightarrow \delta+\varepsilon$ at $436{ }^{\circ} \mathrm{C}$ and 22.3 at\% $\mathrm{Sb}$ based on micrographic data. However, Hansen [14] and later Massalski [3] did not consider the work of Heumann and Heinemann in their assessments, instead establishing the eutectoid decomposition $\varepsilon \rightarrow \delta+\eta$ at $375{ }^{\circ} \mathrm{C}$, which was also determined by micrographic data from Heumann

Table 1 Temperature-invariant reactions in the $\mathrm{Cu}-\mathrm{Sb}$ system [1]

\begin{tabular}{lcccll}
\hline Reaction & \multicolumn{3}{c}{ Composition /at\% Sb } & Temp. $/{ }^{\circ} \mathrm{C}$ & Reaction type \\
\hline $\mathrm{L} \rightarrow \beta$ & & 29 & & 683 & Congruent melt \\
$\mathrm{L} \rightarrow(\mathrm{Cu})+\beta$ & 5.8 & 19 & 19.5 & 645 & Eutectic \\
$\mathrm{L}+\beta \rightarrow \eta$ & 31 & 33.5 & 46 & 586 & Peritectic \\
$\mathrm{L} \rightarrow \eta+(\mathrm{Sb})$ & 33.5 & 63 & 99.9 & 526 & Eutectic \\
$(\mathrm{Cu})+\beta \rightarrow \gamma$ & 5.65 & 15.5 & 21.5 & 488 & Peritectoid \\
$\beta+\gamma \rightarrow \delta$ & 16.5 & 19 & 24 & 462 & Peritectoid \\
$\beta+\delta \rightarrow \varepsilon$ & 19.5 & 23 & 25.5 & $445^{\mathrm{a}}$ & Peritectoid \\
$\beta \rightarrow \varepsilon+\eta$ & 25.5 & 26.5 & 32 & $440^{\mathrm{a}}$ & Eutectoid \\
$\gamma \rightarrow(\mathrm{Cu})+\delta$ & 4.6 & 15.5 & 18.5 & 400 & Eutectoid \\
$\delta+\varepsilon \rightarrow \zeta$ & 20 & 21.5 & 23.5 & $390^{\mathrm{a}}$ & Peritectoid \\
$\varepsilon \rightarrow \zeta+\eta$ & 22.5 & 24 & 32.5 & 360 & Eutectoid \\
$\zeta \rightarrow \delta+\eta$ & 20 & 21.5 & 32.5 & $260^{\mathrm{a}}$ & Eutectoid \\
\hline
\end{tabular}

a Uncertain values
Fig. 1 Current version of the phase diagram of the $\mathrm{Cu}-\mathrm{Sb}$ system [3]

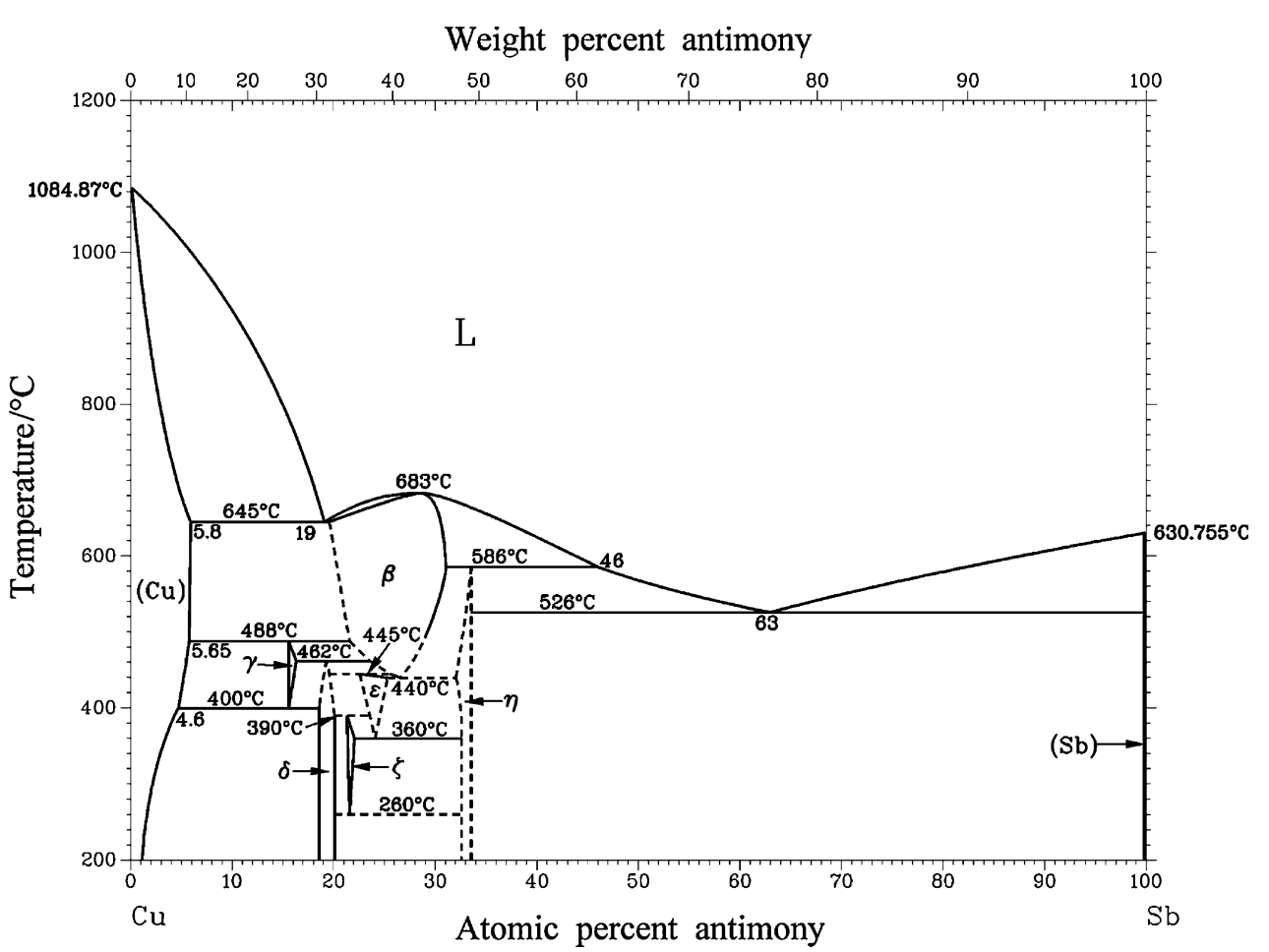


Table 2 Crystallographic data for $\mathrm{Cu}-\mathrm{Sb}$ phases

\begin{tabular}{|c|c|c|c|c|c|c|c|c|c|}
\hline Phase & Stoichiometry & Type & Pearson symbol & Space group & No. & $a / \AA$ & $b / \AA$ & $c / \AA$ & Ref. \\
\hline$\alpha$ & $(\mathrm{Cu})$ & $\mathrm{Cu}$ & $\mathrm{cF} 4$ & $F m-3 m$ & 225 & 3.6130 & - & - & [4] \\
\hline$\beta$ & $\mathrm{Cu}_{3} \mathrm{Sb}$ & $\mathrm{BiF}_{3}$ & $\mathrm{cF} 16$ & $F m-3 m$ & 225 & 6.0000 & - & - & {$[5]$} \\
\hline$\gamma$ & $\mathrm{Cu}_{4} \mathrm{Sb}$ & $\mathrm{Mg}$ & $\mathrm{hP} 2$ & P63/mmc & 194 & 2.7520 & - & 4.3200 & {$[6]$} \\
\hline$\delta$ & $\mathrm{Cu}_{78} \mathrm{Sb}_{20}$ & $\mathrm{Cu}_{78} \mathrm{Sb}_{21}$ & hP98 & P63/mmc & 194 & 19.124 & - & 4.324756 & [7] \\
\hline$\varepsilon$ & $\mathrm{Cu}_{3} \mathrm{Sb}$ & $\mathrm{Cu}_{3} \mathrm{Ti}$ & oP8 & Pmmn & 59 & 5.5040 & 4.3530 & 4.7680 & [8] \\
\hline$\zeta$ & $\mathrm{Cu}_{10} \mathrm{Sb}_{3}$ & $\mathrm{Cu}_{10} \mathrm{Sb}_{3}$ & hP26 & $P-3$ & 147 & 9.9200 & - & 4.3200 & [9] \\
\hline$\eta$ & $\mathrm{Cu}_{2} \mathrm{Sb}$ & $\mathrm{Cu}_{2} \mathrm{Sb}$ & tP6 & P4/nmm & 129 & 4.0014 & - & 6.1044 & [10] \\
\hline$\theta$ & (Sb) & As & hR2 & $R-3 m$ & 166 & 4.3084 & - & 11.2740 & [11] \\
\hline
\end{tabular}

and Heinemann's work [13]. Later, Günzel and Schubert [15] described a new phase $(\zeta)$ occurring on the Sb-rich side of $\delta$. Therefore, the latter reaction had to be corrected to $\varepsilon \rightarrow \zeta+\eta$ (adjusted from 375 to $360{ }^{\circ} \mathrm{C}$ ). The $\gamma$ phase is formed from the $\beta$ phase with $(\mathrm{Cu})$ in the peritectoid reaction $(\mathrm{Cu})+\beta \rightarrow \gamma\left(488^{\circ} \mathrm{C}\right)$. This transformation and the peritectoid reaction $\gamma+\beta \rightarrow \delta\left(462{ }^{\circ} \mathrm{C}\right)$ were found by Murakami and Shibata [16], and both were confirmed by Schubert and Ilschner [12] using dilatometric methods. The $\varepsilon$ phase was first mentioned by the same authors. They tentatively fixed the respective reaction temperatures and the concentration limits according to their high-temperature $\mathrm{X}$-ray diffraction results. The invariant peritectoid temperatures of the reactions $\beta+\delta \rightarrow \varepsilon\left(445^{\circ} \mathrm{C}\right)$ and $\delta+\varepsilon \rightarrow \zeta\left(390{ }^{\circ} \mathrm{C}\right)$ as well as the eutectoid decomposition of $\zeta\left(\zeta \rightarrow \delta+\eta, 280{ }^{\circ} \mathrm{C}\right)$ were only roughly estimated by Günzel and Schubert [15] from X-ray diffraction experiments. They proposed the peritectoid temperature $(\delta+\varepsilon \rightarrow \zeta)$ to be $390{ }^{\circ} \mathrm{C}$, but due to the scatter in their experimental data it can only be said to occur in the temperature range $375-400{ }^{\circ} \mathrm{C}$. The decomposition temperature $(\zeta \rightarrow \delta+\eta)$ is suggested to be $260{ }^{\circ} \mathrm{C}$, but again this temperature can only be stated to lie between 250 and $300{ }^{\circ} \mathrm{C}$. The experimental evidence for the eutectoid reaction $\gamma \rightarrow(\mathrm{Cu})+\delta$ at $400{ }^{\circ} \mathrm{C}$ as presented in the phase diagrams of Hansen [14] and Massalski [3] is unknown. Thus, some reaction temperatures and phase homogeneity ranges are tentative and not determined precisely yet. This is shown by dashed lines in the assessment of the $\mathrm{Cu}-\mathrm{Sb}$ system by Massalski [3]. Further works by Liu et al. [2] in 2000 and Gierlotka et al. [1] in 2009 contribute thermodynamic assessments with similar transition temperatures to those described by Massalski [3]. These works are the most recent ones; nevertheless, information on the ranges of homogeneity of many phases is missing. Liu et al. [2] modeled the liquid, the $(\mathrm{Cu})$, the $(\mathrm{Sb})$, and the $\beta$ phases as solid solutions, as did Gierlotka et al. [1], but the latter also calculated the $\delta$ and the $\gamma$ phases as sublattice models. The results obtained in the present work are compared with those given in [3].

\section{Results and discussion}

The samples used for DTA measurements were annealed for four weeks at $340{ }^{\circ} \mathrm{C}$ or six months at $170{ }^{\circ} \mathrm{C}$ and quenched in cold water. The temperature program included two heating and cooling loops, starting from the annealing temperature and ending $50-100{ }^{\circ} \mathrm{C}$ above the estimated liquidus temperature. The heating rate was $5 \mathrm{~K} / \mathrm{min}$, the measured temperatures are summarized in Table 3 , the DTA curves can be found in Fig. 2, and the corresponding invariant reactions are listed in Table 4 . In addition, we generally performed measurements with heating rates of $10 \mathrm{~K} / \mathrm{min}$ in order to observe the influence of the heating rate on the characteristic temperatures. There was no significant change in the transition temperatures when the heating rate was increased. The temperatures of the maxima of the melting peaks of all samples are consistent with the liquidus temperatures given in [3]. The solidus of the $\beta$ phase, which was established by performing DTA measurements of five samples with $21-28$ at $\% \mathrm{Sb}$, was also in agreement with the literature [3]. The reaction temperature as well as the liquidus concentration of 19 at $\% \mathrm{Sb}$ for the eutectic reaction located at $645{ }^{\circ} \mathrm{C}(\mathrm{L} \rightarrow(\mathrm{Cu})+\beta)$ were confirmed based on three of our samples; see Table 3 . However, samples at 10, 17.5, and 19.5 at $\% \mathrm{Sb}$ showed some discrepancies from the data in the literature at temperatures below $645^{\circ} \mathrm{C}$ [3]. Strong effects were observed in all three samples at temperatures of 467 and $484{ }^{\circ} \mathrm{C}$. We allocated the effect at $467{ }^{\circ} \mathrm{C}$ to the reaction $\beta+\gamma \rightarrow \delta$, which is described in the literature as occurring at $462{ }^{\circ} \mathrm{C}$ [3], and the effect at $484{ }^{\circ} \mathrm{C}$ to $(\mathrm{Cu})+\beta \rightarrow \gamma$ (which takes place at $488{ }^{\circ} \mathrm{C}$ according to the literature [3]). However, according to the phase relations [3], the effect at $467{ }^{\circ} \mathrm{C}$ should not be observable in the sample with 10 at $\% \mathrm{Sb}$ in the first heating run. Surprisingly, this effect was even stronger in the second heating run. In order to clarify this discrepancy, we annealed this sample at 470 and $480{ }^{\circ} \mathrm{C}$ for 28 days. Both temperatures resulted in large amounts of (Cu) and $\gamma$, but also traces of the $\beta$ phase (see Tables 5, 6). It is worth noting at this point that the $\beta$ phase cannot be 
Table 3 Summary of measured thermal effects

\begin{tabular}{|c|c|c|c|c|c|c|}
\hline \multirow[t]{3}{*}{ No. } & \multirow[t]{3}{*}{ Nominal comp. /at $\%$} & \multirow[t]{3}{*}{ Heat treatment } & \multicolumn{4}{|l|}{ Thermal analysis } \\
\hline & & & \multicolumn{3}{|l|}{ Heating $/{ }^{\circ} \mathrm{C}$} & \multirow{2}{*}{$\begin{array}{l}\text { Cooling } /{ }^{\circ} \mathrm{C} \\
\text { Liquidus }\end{array}$} \\
\hline & & & Invariant effects & Other effects & Liquidus & \\
\hline 1 & $\mathrm{Cu}_{90} \mathrm{Sb}_{10}$ & $340{ }^{\circ} \mathrm{C}, 28$ days & $469,482.2,642.2$ & & 926.1 & 920.6 \\
\hline 2 & $\mathrm{Cu}_{82.5} \mathrm{Sb}_{17.5}$ & $340{ }^{\circ} \mathrm{C}, 28$ days & $440,467.1,485,644.9$ & & 651.5 & 642.2 \\
\hline 3 & $\mathrm{Cu}_{80.5} \mathrm{Sb}_{19.5}$ & $340{ }^{\circ} \mathrm{C}, 28$ days & $461.8,486.8$ & 641.8 & 650.9 & 641.4 \\
\hline 4 & $\mathrm{Cu}_{79} \mathrm{Sb}_{21}$ & $340{ }^{\circ} \mathrm{C}, 28$ days & $443.5,451.9$ & 644.2 & 654.6 & 647.5 \\
\hline 5 & $\mathrm{Cu}_{77.5} \mathrm{Sb}_{22.5}$ & $340{ }^{\circ} \mathrm{C}, 28$ days & $360.5,441.4$ & 648.9 & 660.8 & 655.2 \\
\hline 6 & $\mathrm{Cu}_{76} \mathrm{Sb}_{24}$ & $170^{\circ} \mathrm{C}, 6$ months & $(323.3)^{\mathrm{a}}, 363.3$ & $436.8,655$ & 670.6 & 665.9 \\
\hline 7 & $\mathrm{Cu}_{74} \mathrm{Sb}_{26}$ & $170^{\circ} \mathrm{C}, 6$ months & $(302.3)^{\mathrm{a}}, 375.8,431.9$ & 668.9 & 681.6 & 675.0 \\
\hline 8 & $\mathrm{Cu}_{72} \mathrm{Sb}_{28}$ & $340{ }^{\circ} \mathrm{C}, 28$ days & 428.6 & $433.8,679.7$ & 690.2 & 679.3 \\
\hline 9 & $\mathrm{Cu}_{70} \mathrm{Sb}_{30}$ & Melt & 426.7 & $470.3,673.1$ & 686.3 & 676.7 \\
\hline 10 & $\mathrm{Cu}_{60} \mathrm{Sb}_{40}$ & $340{ }^{\circ} \mathrm{C}, 28$ days & $525.8,586.2$ & & 616.0 & 597.3 \\
\hline 11 & $\mathrm{Cu}_{35} \mathrm{Sb}_{65}$ & $340{ }^{\circ} \mathrm{C}, 28$ days & 524.8 & & 539.9 & 506.6 \\
\hline 12 & $\mathrm{Cu}_{30} \mathrm{Sb}_{70}$ & $340{ }^{\circ} \mathrm{C}, 28$ days & 525.4 & & 546.4 & 518.2 \\
\hline 13 & $\mathrm{Cu}_{25} \mathrm{Sb}_{75}$ & $340{ }^{\circ} \mathrm{C}, 28$ days & 526.5 & & 553.9 & 517.7 \\
\hline
\end{tabular}

a Very weak effect

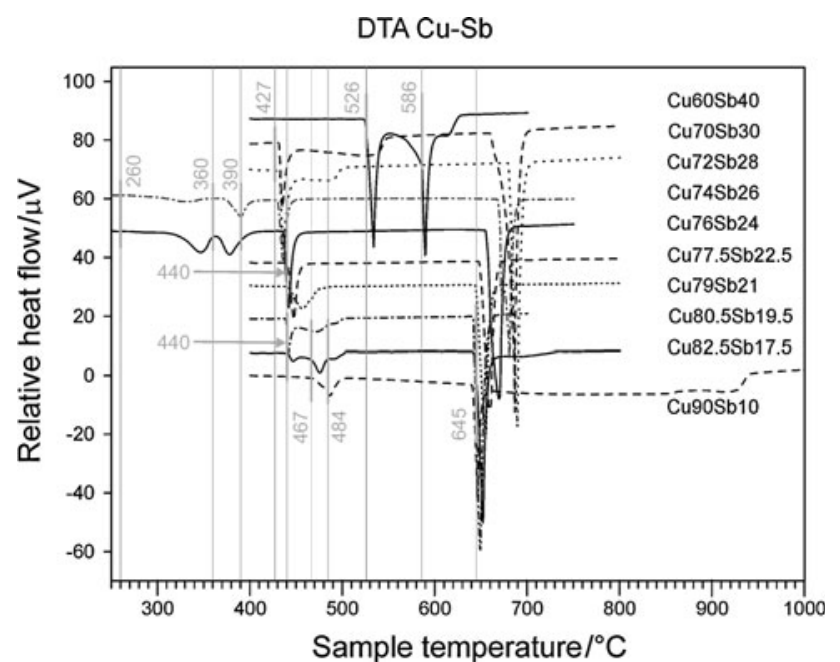

Fig. 2 DTA curves of samples with $10-40$ at $\% \mathrm{Sb}$

quenched; it mainly decomposes to the low-temperature phases $\delta$ and $\varepsilon$. Thus, we instead assume that $(\mathrm{Cu})$ is in equilibrium with $\gamma$ at both temperatures. Although the effect is clearly present at $467{ }^{\circ} \mathrm{C}$ in the sample with 10 at $\% \mathrm{Sb}$, we have decided not to change the previously accepted phase diagram given in the literature [3]. XRD analysis of $\mathrm{Cu}_{90} \mathrm{Sb}_{10}$ annealed at $435{ }^{\circ} \mathrm{C}$ and $\mathrm{Cu}_{82.5} \mathrm{Sb}_{17.5}$ annealed at $430{ }^{\circ} \mathrm{C}$ showed $(\mathrm{Cu})$ and $\delta$ as equilibrium phases (see Fig. 3). According to the literature, these samples should both contain the $\gamma$ phase [3]. Supported by an invariant reaction observed at $440{ }^{\circ} \mathrm{C}$ during DTA of $\mathrm{Cu}_{82.5} \mathrm{Sb}_{17.5}$, we fixed the eutectoid reaction $\gamma \rightarrow(\mathrm{Cu})+\delta$ at this temperature. This is additionally supported by the fact that the original source of the reaction temperature of $400{ }^{\circ} \mathrm{C}$ given in [3] could not be found and thus appears to be estimated. The peritectoid reaction $\beta+\delta \rightarrow \varepsilon$ was corroborated by DTA of samples with 21 and 22.5 at $\% \mathrm{Sb}$. However, the corresponding temperature $\left(440{ }^{\circ} \mathrm{C}\right)$ differs slightly from the literature value $\left(445{ }^{\circ} \mathrm{C}\right.$ [3]). DTA of these samples should also show invariant reactions according to $\varepsilon+\delta \rightarrow \zeta\left(390^{\circ} \mathrm{C}\right)$ and $\varepsilon \rightarrow \zeta+\eta\left(360{ }^{\circ} \mathrm{C}\right)$, and we did indeed find the reaction at $360^{\circ} \mathrm{C}$ in $\mathrm{Cu}_{87.5} \mathrm{Sb}_{22.5}$ as a weak effect in the second heating run. However, we could not locate the peritectoid reaction at $390{ }^{\circ} \mathrm{C}$. Thermal analysis of the samples with 24 and 26 at\% $\mathrm{Sb}$ agreed well with the previously reported phase diagram [3] above $350{ }^{\circ} \mathrm{C}$. On the other hand, DTA of samples annealed at $170{ }^{\circ} \mathrm{C}$ did not indicate the invariant reaction at $260{ }^{\circ} \mathrm{C} \zeta \rightarrow \delta+\eta$. Instead, we found two further signals at different temperatures that are possibly related to this reaction $\left(24\right.$ at $\% \mathrm{Sb} 323{ }^{\circ} \mathrm{C}, 26$ at $\% \mathrm{Sb}$ $302{ }^{\circ} \mathrm{C}$; see also Table 3). Because XRD data for the samples with $21,22.5,24$, and 26 at $\% \mathrm{Sb}$ are consistent with the literature data [3], we kept the previously reported phase relations and reaction temperatures. Using the samples with 28 and 30 at\% $\mathrm{Sb}$, we were able to determine the temperature of the eutectoid reaction $\beta \rightarrow \varepsilon+\eta$ as $427{ }^{\circ} \mathrm{C}$, which had previously been estimated as $440{ }^{\circ} \mathrm{C}$ ([3]: dashed lines, see Fig. 1). The liquidus and solidus curves allowed us to estimate the congruent melting point of the $\beta$ phase at $690{ }^{\circ} \mathrm{C}$ and 29 at $\% \mathrm{Sb}\left([3], 683{ }^{\circ} \mathrm{C}\right)$. Finally, we also verified the eutectic reaction at $526{ }^{\circ} \mathrm{C}$ $(\mathrm{L} \rightarrow \eta+(\mathrm{Sb}))$ and the peritectic reaction at $586{ }^{\circ} \mathrm{C}$ $(\beta+\mathrm{L} \rightarrow \eta)$. 
Table 4 Comparison of reactions and temperatures published in the literature and found in this work

\begin{tabular}{llll}
\hline Invariant reactions & Temp. $/{ }^{\circ} \mathrm{C}:[1]$ & Temp. $/{ }^{\circ} \mathrm{C}:$ this work & Comments \\
\hline $\mathrm{L} \rightarrow \beta$ & 683 & 690 & Estimated from liquidus curves \\
$\mathrm{L} \rightarrow(\mathrm{Cu})+\beta$ & 645 & 645 & \\
$\mathrm{~L}+\beta \rightarrow \eta$ & 586 & 586 & \\
$\mathrm{~L} \rightarrow \eta+(\mathrm{Sb})$ & 526 & 526 & \\
$(\mathrm{Cu})+\beta \rightarrow \gamma$ & 488 & 484 & \\
$\beta+\gamma \rightarrow \delta$ & 462 & 467 & Not detected, adopted from literature \\
$\beta+\delta \rightarrow \varepsilon$ & 445 & 440 & Not detected, adopted from literature \\
$\beta \rightarrow \varepsilon+\eta$ & 440 & 427 & \\
$\gamma \rightarrow(\mathrm{Cu})+\delta$ & 400 & 440 & \\
$\delta+\varepsilon \rightarrow \zeta$ & 390 & 390 & 360 \\
$\varepsilon \rightarrow \zeta+\eta$ & 360 & 260 & \\
$\zeta \rightarrow \delta+\eta$ & 260 & &
\end{tabular}

Table 5 Crystal structures and lattice parameters of quenched $\mathrm{Cu}-\mathrm{Sb}$ samples

\begin{tabular}{|c|c|c|c|c|c|}
\hline Sample & Heat treatment & Phase & $\begin{array}{l}\text { Structure } \\
\text { type }\end{array}$ & Lattice parameter $/ \AA$ & Comment \\
\hline \multirow[t]{21}{*}{$\mathrm{Cu}_{90} \mathrm{Sb}_{10}$} & \multirow[t]{2}{*}{ Melt $1,000{ }^{\circ} \mathrm{C}, 1$ day } & $\alpha=(\mathrm{Cu})$ & $F m-3 m$ & $a=3.662(4)$ & \multirow[t]{12}{*}{ Nonequilibrium (quenched from liquid) } \\
\hline & & $\delta=\mathrm{Cu}_{78} \mathrm{Sb}_{20}$ & $P 6_{3} / m m c$ & $a=19.035(3), c=4.3285(9)$ & \\
\hline & \multirow[t]{2}{*}{$340{ }^{\circ} \mathrm{C}, 28$ days } & $\alpha=(\mathrm{Cu})$ & $F m-3 m$ & $a=3.64479(2)$ & \\
\hline & & $\delta=\mathrm{Cu}_{78} \mathrm{Sb}_{20}$ & $\mathrm{P}_{3} / \mathrm{mmc}$ & $a=19.0823(2), c=4.32615(9)$ & \\
\hline & \multirow[t]{2}{*}{$390^{\circ} \mathrm{C}, 28$ days } & $\alpha=(\mathrm{Cu})$ & $F m-3 m$ & $a=3.65393(6)$ & \\
\hline & & $\delta=\mathrm{Cu}_{78} \mathrm{Sb}_{20}$ & $P 6_{3} / m m c$ & $a=19.0421(4), c=4.3260(2)$ & \\
\hline & \multirow[t]{2}{*}{$420^{\circ} \mathrm{C}, 28$ days } & $\alpha=(\mathrm{Cu})$ & $F m-3 m$ & $a=3.66266(7)$ & \\
\hline & & $\delta=\mathrm{Cu}_{78} \mathrm{Sb}_{20}$ & $P 6_{3} / m m c$ & $a=19.0286(5), c=4.3291(2)$ & \\
\hline & \multirow[t]{2}{*}{$435^{\circ} \mathrm{C}, 28$ days } & $\alpha=(\mathrm{Cu})$ & $F m-3 m$ & $a=3.66487(9)$ & \\
\hline & & $\delta=\mathrm{Cu}_{78} \mathrm{Sb}_{20}$ & $\mathrm{Po}_{3} / m m c$ & $a=19.0074(4), c=4.3281(1)$ & \\
\hline & \multirow[t]{2}{*}{$450{ }^{\circ} \mathrm{C}, 28$ days } & $\alpha=(\mathrm{Cu})$ & $F m-3 m$ & $a=3.6686(1)$ & \\
\hline & & $\gamma=\mathrm{Cu}_{4} \mathrm{Sb}$ & $\mathrm{P}_{3} / \mathrm{mmc}$ & $a=2.68573(9), c=4.3274(3)$ & \\
\hline & \multirow[t]{3}{*}{$470{ }^{\circ} \mathrm{C}, 28$ days } & $\alpha=(\mathrm{Cu})$ & $F m-3 m$ & $a=3.62263(2)$ & \multirow[t]{3}{*}{$\beta$ phase partially stabilized } \\
\hline & & $\beta=\mathrm{Cu}_{3} \mathrm{Sb}$ & $F m-3 m$ & $a=5.8105(1)$ & \\
\hline & & $\gamma=\mathrm{Cu}_{4} \mathrm{Sb}$ & $P 6_{3} / m m c$ & $a=3.3521(3), c=2.8928(5)$ & \\
\hline & \multirow[t]{3}{*}{$480^{\circ} \mathrm{C}, 28$ days } & $\alpha=(\mathrm{Cu})$ & $F m-3 m$ & $a=3.6719(1)$ & \multirow[t]{6}{*}{$\beta$ phase partially stabilized } \\
\hline & & $\beta=\mathrm{Cu}_{3} \mathrm{Sb}$ & $F m-3 m$ & $a=5.9162(9)$ & \\
\hline & & $\gamma=\mathrm{Cu}_{4} \mathrm{Sb}$ & $\mathrm{P}_{3} / \mathrm{mmc}$ & $a=2.7527(4), c=4.244(2)$ & \\
\hline & \multirow[t]{3}{*}{$600{ }^{\circ} \mathrm{C}, 28$ days } & $\alpha=(\mathrm{Cu})$ & $F m-3 m$ & $a=3.67907(7)$ & \\
\hline & & $\gamma=\mathrm{Cu}_{4} \mathrm{Sb}$ & $\mathrm{P}_{3} / m m c$ & $a=2.74168(8), c=4.3304(2)$ & \\
\hline & & $\delta=\mathrm{Cu}_{78} \mathrm{Sb}_{20}$ & $P 6_{3} / m m c$ & $a=19.101(3), c=4.3307(8)$ & \\
\hline \multirow[t]{11}{*}{$\mathrm{Cu}_{82.5} \mathrm{Sb}_{17.5}$} & \multirow[t]{2}{*}{ Melt $1,000^{\circ} \mathrm{C}, 1$ day } & $\delta=\mathrm{Cu}_{78} \mathrm{Sb}_{20}$ & $P 6_{3} / m m c$ & $a=19.1198(4), c=4.3273(1)$ & \multirow[t]{8}{*}{ Nonequilibrium (quenched from liquid) } \\
\hline & & $\varepsilon=\mathrm{Cu}_{3} \mathrm{Sb}$ & Pmmn & $a=5.5045(3), b=4.3355(2), c=4.7549(4)$ & \\
\hline & \multirow[t]{2}{*}{$340{ }^{\circ} \mathrm{C}, 28$ days } & $\alpha=(\mathrm{Cu})$ & $F m-3 m$ & $a=3.6482(9)$ & \\
\hline & & $\delta=\mathrm{Cu}_{78} \mathrm{Sb}_{20}$ & $\mathrm{P}_{3} / \mathrm{mmc}$ & $a=19.0836(1), c=4.32763(4)$ & \\
\hline & \multirow[t]{2}{*}{$430^{\circ} \mathrm{C}, 28$ days } & $\alpha=(\mathrm{Cu})$ & $F m-3 m$ & $a=3.6676(7)$ & \\
\hline & & $\delta=\mathrm{Cu}_{78} \mathrm{Sb}_{20}$ & $P 6_{3} / m m c$ & $a=18.9911(2), c=4.32639(8)$ & \\
\hline & \multirow[t]{2}{*}{$470{ }^{\circ} \mathrm{C}, 28$ days } & $\gamma=\mathrm{Cu}_{4} \mathrm{Sb}$ & $\mathrm{PG}_{3} / \mathrm{mmc}$ & $a=2.69586(9), c=4.3309(3)$ & \\
\hline & & $\delta=\mathrm{Cu}_{78} \mathrm{Sb}_{20}$ & $\mathrm{P}_{3} / m m c$ & $a=19.0113(5), c=4.3278(2)$ & \\
\hline & \multirow[t]{3}{*}{$600{ }^{\circ} \mathrm{C}, 28$ days } & $\alpha=(\mathrm{Cu})$ & $F m-3 m$ & $a=3.6805(4)$ & \multirow[t]{3}{*}{$\beta$ phase partially stabilized } \\
\hline & & $\beta=\mathrm{Cu}_{3} \mathrm{Sb}$ & $F m-3 m$ & $a=5.9239(5)$ & \\
\hline & & $\delta=\mathrm{Cu}_{78} \mathrm{Sb}_{20}$ & $\mathrm{P}_{3} / \mathrm{mmc}$ & $a=19.1485(3), c=4.3291(1)$ & \\
\hline
\end{tabular}


Table 5 continued

\begin{tabular}{|c|c|c|c|c|c|}
\hline Sample & Heat treatment & Phase & Structure type & Lattice parameter $/ \AA$ & Comment \\
\hline $\mathrm{Cu}_{80.5} \mathrm{Sb}_{19.5}$ & $340{ }^{\circ} \mathrm{C}, 28$ days & $\delta=\mathrm{Cu}_{78} \mathrm{Sb}_{20}$ & $P 6_{3} / m m c$ & $a=19.1178(1), c=4.32590(6)$ & \\
\hline \multirow[t]{9}{*}{$\mathrm{Cu}_{79.5} \mathrm{Sb}_{20.5}$} & \multirow[t]{3}{*}{$170{ }^{\circ} \mathrm{C}, 6$ months } & $\delta=\mathrm{Cu}_{78} \mathrm{Sb}_{20}$ & $\mathrm{PG}_{3} / \mathrm{mmc}$ & $a=19.1241(5), c=4.3279(1)$ & \multirow{9}{*}{$\begin{array}{l}\text { Nonequilibrium (not } \\
\text { sufficiently annealed) }\end{array}$} \\
\hline & & $\zeta=\mathrm{Cu}_{10} \mathrm{Sb}_{3}$ & $P-3$ & $a=9.9335(1), c=4.3227(1)$ & \\
\hline & & $\eta=\mathrm{Cu}_{2} \mathrm{Sb}$ & $P 4 / n m m$ & $a=4.0035(7), c=6.087(2)$ & \\
\hline & \multirow[t]{2}{*}{$280{ }^{\circ} \mathrm{C}, 28$ days } & $\delta=\mathrm{Cu}_{78} \mathrm{Sb}_{20}$ & $\mathrm{PG}_{3} / m m c$ & $a=19.1476(2), c=4.32586(9)$ & \\
\hline & & $\zeta=\mathrm{Cu}_{10} \mathrm{Sb}_{3}$ & $P-3$ & $a=9.90716(7), c=4.32301(8)$ & \\
\hline & \multirow[t]{2}{*}{$350{ }^{\circ} \mathrm{C}, 28$ days } & $\delta=\mathrm{Cu}_{78} \mathrm{Sb}_{20}$ & $\mathrm{PG}_{3} / m m c$ & $a=19.1604(4), c=4.3249(1)$ & \\
\hline & & $\zeta=\mathrm{Cu}_{10} \mathrm{Sb}_{3}$ & $P-3$ & $a=9.89815(8), c=4.32278(6)$ & \\
\hline & \multirow[t]{2}{*}{$420{ }^{\circ} \mathrm{C}, 28$ days } & $\delta=\mathrm{Cu}_{78} \mathrm{Sb}_{20}$ & $\mathrm{PG}_{3} / m m c$ & $a=19.1655(1), c=4.32620(3)$ & \\
\hline & & $\varepsilon=\mathrm{Cu}_{3} \mathrm{Sb}$ & Pmmn & $a=5.493(2), b=4.3468(2), c=4.757(2)$ & \\
\hline \multirow[t]{8}{*}{$\mathrm{Cu}_{79} \mathrm{Sb}_{21}$} & \multirow[t]{2}{*}{ Melt $1,000{ }^{\circ} \mathrm{C}, 1$ day } & $\delta=\mathrm{Cu}_{78} \mathrm{Sb}_{20}$ & $\mathrm{~Pb}_{3} / m m c$ & $a=19.1665(8), c=4.3317(3)$ & \multirow{5}{*}{$\begin{array}{l}\text { Nonequilibrium (quenched } \\
\text { from liquid) }\end{array}$} \\
\hline & & $\varepsilon=\mathrm{Cu}_{3} \mathrm{Sb}$ & Pmmn & $a=5.4977(3), b=4.3301(1), c=4.7698(2)$ & \\
\hline & $340{ }^{\circ} \mathrm{C}, 28$ days & $\zeta=\mathrm{Cu}_{10} \mathrm{Sb}_{3}$ & $P-3$ & $a=9.90817(7), c=4.32364(5)$ & \\
\hline & \multirow[t]{2}{*}{$430{ }^{\circ} \mathrm{C}, 28$ days } & $\delta=\mathrm{Cu}_{78} \mathrm{Sb}_{20}$ & $\mathrm{PG}_{3} / m m c$ & $a=19.1082(2), c=4.32665(9)$ & \\
\hline & & $\varepsilon=\mathrm{Cu}_{3} \mathrm{Sb}$ & Pmmn & $a=5.4920(2), b=4.34468(8), c=4.7510(2)$ & \\
\hline & \multirow[t]{3}{*}{$470{ }^{\circ} \mathrm{C}, 28$ days } & $\beta=\mathrm{Cu}_{3} \mathrm{Sb}$ & $F m-3 m$ & $a=6.0108(8)$ & \multirow[t]{3}{*}{$\beta$ phase partially stabilized } \\
\hline & & $\delta=\mathrm{Cu}_{78} \mathrm{Sb}_{20}$ & $\mathrm{PC}_{3} / m m c$ & $a=19.1990(2), c=4.33742(8)$ & \\
\hline & & $\varepsilon=\mathrm{Cu}_{3} \mathrm{Sb}$ & Pmmn & $a=5.443(1), b=4.3296(3), c=4.711(1)$ & \\
\hline \multirow[t]{15}{*}{$\mathrm{Cu}_{77.5} \mathrm{Sb}_{22.5}$} & \multirow[t]{2}{*}{ Melt $1,000{ }^{\circ} \mathrm{C}, 1$ day } & $\delta=\mathrm{Cu}_{78} \mathrm{Sb}_{20}$ & $P 6_{3} / m m c$ & $a=19.2303(6), c=4.3342(2)$ & \multirow{6}{*}{$\begin{array}{l}\text { Nonequilibrium (quenched } \\
\text { from liquid) }\end{array}$} \\
\hline & & $\varepsilon=\mathrm{Cu}_{3} \mathrm{Sb}$ & Pmmn & $a=5.5054(5), b=4.3409(2), c=4.7631(5)$ & \\
\hline & \multirow[t]{2}{*}{$340{ }^{\circ} \mathrm{C}, 28$ days } & $\zeta=\mathrm{Cu}_{10} \mathrm{Sb}_{3}$ & $P-3$ & $a=9.92390(6), c=4.32223(5)$ & \\
\hline & & $\eta=\mathrm{Cu}_{2} \mathrm{Sb}$ & $P 4 / n m m$ & $a=4.0018(2), c=6.1031(5)$ & \\
\hline & \multirow[t]{2}{*}{$430{ }^{\circ} \mathrm{C}, 28$ days } & $\delta=\mathrm{Cu}_{78} \mathrm{Sb}_{20}$ & $\mathrm{PG}_{3} / m m c$ & $a=19.156(1), c=4.3273(4)$ & \\
\hline & & $\varepsilon=\mathrm{Cu}_{3} \mathrm{Sb}$ & Pmmn & $a=5.49427(9), b=4.34601(5), c=4.75169(9)$ & \\
\hline & \multirow[t]{3}{*}{$450{ }^{\circ} \mathrm{C}, 28$ days } & $\delta=\mathrm{Cu}_{78} \mathrm{Sb}_{20}$ & $P 6_{3} / m m c$ & $a=19.022(5), c=4.4541(9)$ & \multirow{3}{*}{$\begin{array}{l}\text { Nonequilibrium: } \beta \text { phase } \\
\text { decomposed during } \\
\text { quenching }\end{array}$} \\
\hline & & $\varepsilon=\mathrm{Cu}_{3} \mathrm{Sb}$ & Pmmn & $a=5.3831(5), b=4.2871(5), c=5.0467(6)$ & \\
\hline & & $\eta=\mathrm{Cu}_{2} \mathrm{Sb}$ & $P 4 / n m m$ & $a=4.2732(3), c=5.7367(7)$ & \\
\hline & \multirow[t]{3}{*}{$470{ }^{\circ} \mathrm{C}, 28$ days } & $\delta=\mathrm{Cu}_{78} \mathrm{Sb}_{20}$ & $\mathrm{PG}_{3} / m m c$ & $a=18.973(4), c=4.4484(8)$ & \multirow{3}{*}{$\begin{array}{l}\text { Nonequilibrium: } \beta \text { phase } \\
\text { decomposed during } \\
\text { quenching }\end{array}$} \\
\hline & & $\varepsilon=\mathrm{Cu}_{3} \mathrm{Sb}$ & Pmmn & $a=5.3763(7), b=4.2818(5), c=5.0382(5)$ & \\
\hline & & $\eta=\mathrm{Cu}_{2} \mathrm{Sb}$ & P4/nmm & $a=4.2675(2), c=5.7335(7)$ & \\
\hline & $600{ }^{\circ} \mathrm{C}, 28$ days & $\delta=\mathrm{Cu}_{78} \mathrm{Sb}_{20}$ & $P 6_{3} / m m c$ & $a=18.992(4), c=4.4519(8)$ & Nonequilibrium: $\beta$ phase \\
\hline & & $\varepsilon=\mathrm{Cu}_{3} \mathrm{Sb}$ & Pmmn & $a=5.3810(9), b=4.2812(6), c=5.0447(6)$ & decomposed during \\
\hline & & $\eta=\mathrm{Cu}_{2} \mathrm{Sb}$ & $P 4 / n m m$ & $a=4.2667(2), c=5.7424(7)$ & \\
\hline $\mathrm{Cu}_{76} \mathrm{Sb}_{24}$ & $170^{\circ} \mathrm{C}, 6$ months & $\delta=\mathrm{Cu}_{78} \mathrm{Sb}_{20}$ & $\mathrm{PG}_{3} / \mathrm{mmc}$ & $a=19.1412(1), c=4.32539(6)$ & \\
\hline & & $\eta=\mathrm{Cu}_{2} \mathrm{Sb}$ & $P 4 / n m m$ & $a=4.00170(4), c=6.1027(1)$ & \\
\hline & $280{ }^{\circ} \mathrm{C}, 28$ days & $\zeta=\mathrm{Cu}_{10} \mathrm{Sb}_{3}$ & $P-3$ & $a=9.92096(5), c=4.32247(4)$ & \\
\hline & & $\eta=\mathrm{Cu}_{2} \mathrm{Sb}$ & P4/nmm & $a=4.00200(4), c=6.1038(1)$ & \\
\hline & $340{ }^{\circ} \mathrm{C}, 28$ days & $\zeta=\mathrm{Cu}_{10} \mathrm{Sb}_{3}$ & $P-3$ & $a=9.92319(6), c=4.32294(4)$ & \\
\hline & & $\eta=\mathrm{Cu}_{2} \mathrm{Sb}$ & P4/nmm & $a=4.00364(5), c=6.1042(2)$ & \\
\hline & $400{ }^{\circ} \mathrm{C}, 28$ days & $\varepsilon=\mathrm{Cu}_{3} \mathrm{Sb}$ & Pmmn & $a=5.5064(3), b=4.35302(4), c=4.7680(2)$ & \\
\hline $\mathrm{Cu}_{74} \mathrm{Sb}_{26}$ & $170{ }^{\circ} \mathrm{C}, 6$ months & $\alpha=(\mathrm{Cu})$ & $F m-3 m$ & $a=3.6216(1)$ & Nonequilibrium (not \\
\hline & & $\delta=\mathrm{Cu}_{78} \mathrm{Sb}_{20}$ & $\mathrm{~Pb}_{3} / \mathrm{mmc}$ & $a=19.1335(5), c=4.3248(1)$ & sufficiently annealed) \\
\hline & & $\zeta=\mathrm{Cu}_{10} \mathrm{Sb}_{3}$ & $P-3$ & $a=9.9149(3), c=4.3213(2)$ & \\
\hline & & $\eta=\mathrm{Cu}_{2} \mathrm{Sb}$ & $P 4 / n m m$ & $a=4.00201(3), c=6.10281(9)$ & \\
\hline & $280^{\circ} \mathrm{C}, 28$ days & $\zeta=\mathrm{Cu}_{10} \mathrm{Sb}_{3}$ & $P-3$ & $a=9.91867(6), c=4.32272(4)$ & \\
\hline & & $\eta=\mathrm{Cu}_{2} \mathrm{Sb}$ & P4/nmm & $a=4.00203(2), c=6.10400(8)$ & \\
\hline & $340{ }^{\circ} \mathrm{C}, 28$ days & $\zeta=\mathrm{Cu}_{10} \mathrm{Sb}_{3}$ & $P-3$ & $a=9.91806(9), c=4.32282(7)$ & \\
\hline & & $\eta=\mathrm{Cu}_{2} \mathrm{Sb}$ & P4/nmm & $a=4.00295(4), c=6.1034(1)$ & \\
\hline & $400{ }^{\circ} \mathrm{C}, 28$ days & $\varepsilon=\mathrm{Cu}_{3} \mathrm{Sb}$ & Pmmn & $a=5.5090(1), b=4.35420(5), c=4.7757(1)$ & \\
\hline & & $\eta=\mathrm{Cu}_{2} \mathrm{Sb}$ & P4/nmm & $a=4.00147(8), c=6.1038(2)$ & \\
\hline
\end{tabular}


Table 5 continued

\begin{tabular}{|c|c|c|c|c|c|}
\hline Sample & Heat treatment & Phase & $\begin{array}{l}\text { Structure } \\
\text { type }\end{array}$ & Lattice parameter $/ \AA$ & Comment \\
\hline \multirow[t]{4}{*}{$\mathrm{Cu}_{72} \mathrm{Sb}_{28}$} & Melt $1,000^{\circ} \mathrm{C}, 1$ day & $\begin{array}{l}\varepsilon=\mathrm{Cu}_{3} \mathrm{Sb} \\
\eta=\mathrm{Cu}_{2} \mathrm{Sb}\end{array}$ & $\begin{array}{l}\text { Pmmn } \\
\text { P4/nmm }\end{array}$ & $\begin{array}{l}a=5.5132(2), b=4.35595(8), \mathrm{c}=4.7800(1) \\
a=4.00218(5), c=6.1043(1)\end{array}$ & $\begin{array}{l}\text { Nonequilibrium (quenched } \\
\text { from liquid) }\end{array}$ \\
\hline & $340{ }^{\circ} \mathrm{C}, 28$ days & $\begin{array}{l}\zeta=\mathrm{Cu}_{10} \mathrm{Sb}_{3} \\
\eta=\mathrm{Cu}_{2} \mathrm{Sb}\end{array}$ & $\begin{array}{l}P-3 \\
P 4 / n m m\end{array}$ & $\begin{array}{l}a=9.9206(1), c=4.3219(1) \\
a=4.00138(3), c=6.1032(1)\end{array}$ & \\
\hline & $430{ }^{\circ} \mathrm{C}, 28$ days & $\begin{array}{l}\varepsilon=\mathrm{Cu}_{3} \mathrm{Sb} \\
\eta=\mathrm{Cu}_{2} \mathrm{Sb}\end{array}$ & $\begin{array}{l}\text { Pmmn } \\
\text { P4/nmm }\end{array}$ & $\begin{array}{l}a=5.5197(2), b=4.36081(9), c=4.7898(2) \\
a=4.00352(4), c=6.1059(1)\end{array}$ & \\
\hline & $600{ }^{\circ} \mathrm{C}, 28$ days & $\begin{array}{l}\beta=\mathrm{Cu}_{3} \mathrm{Sb} \\
\varepsilon=\mathrm{Cu}_{3} \mathrm{Sb} \\
\eta=\mathrm{Cu}_{2} \mathrm{Sb}\end{array}$ & $\begin{array}{l}\text { Fm-3m } \\
\text { Pmmn } \\
\text { P4/nmm }\end{array}$ & $\begin{array}{l}a=5.9979(9) \\
a=5.3919(8), b=4.2688(7), c=5.0588(7) \\
a=4.00016(7), c=6.0977(2)\end{array}$ & $\beta$ phase partially stabilized \\
\hline \multirow[t]{3}{*}{$\mathrm{Cu}_{70} \mathrm{Sb}_{30}$} & $430{ }^{\circ} \mathrm{C}, 28$ days & $\begin{array}{l}\varepsilon=\mathrm{Cu}_{3} \mathrm{Sb} \\
\eta=\mathrm{Cu}_{2} \mathrm{Sb}\end{array}$ & $\begin{array}{l}\text { Pmmn } \\
\text { P4/nmm }\end{array}$ & $\begin{array}{l}a=5.5157(2), b=4.35768(8), c=4.7830(1) \\
a=4.00130(3), c=6.10265(8)\end{array}$ & \\
\hline & $470{ }^{\circ} \mathrm{C}, 28$ days & $\begin{array}{l}\beta=\mathrm{Cu}_{3} \mathrm{Sb} \\
\varepsilon=\mathrm{Cu}_{3} \mathrm{Sb} \\
\eta=\mathrm{Cu}_{2} \mathrm{Sb}\end{array}$ & $\begin{array}{l}\text { Fm-3m } \\
\text { Pmmn } \\
\text { P4/nmm }\end{array}$ & $\begin{array}{l}a=6.0384(9) \\
a=5.5113(5), b=4.3558(2), c=4.7796(4) \\
a=4.00302(2), c=6.10545(7)\end{array}$ & $\beta$ phase partially stabilized \\
\hline & $600{ }^{\circ} \mathrm{C}, 28$ days & $\begin{array}{l}\beta=\mathrm{Cu}_{3} \mathrm{Sb} \\
\varepsilon=\mathrm{Cu}_{3} \mathrm{Sb} \\
\eta=\mathrm{Cu}_{2} \mathrm{Sb}\end{array}$ & $\begin{array}{l}\text { Fm-3m } \\
\text { Pmmn } \\
\text { P4/nmm }\end{array}$ & $\begin{array}{l}a=6.035(3) \\
a=5.5039(3), b=4.3540(1), c=4.7787(2) \\
a=4.00170(3), c=6.10306(8)\end{array}$ & $\beta$ phase partially stabilized \\
\hline \multirow[t]{4}{*}{$\mathrm{Cu}_{60} \mathrm{Sb}_{40}$} & Melt $1000^{\circ} \mathrm{C}, 1$ day & $\begin{array}{l}\eta=\mathrm{Cu}_{2} \mathrm{Sb} \\
\theta=(\mathrm{Sb})\end{array}$ & $\begin{array}{l}P 4 / n m m \\
R-3 m\end{array}$ & $\begin{array}{l}a=4.00231(2), c=6.10442(8) \\
a=4.3060(1), c=11.2701(7)\end{array}$ & $\begin{array}{l}\text { Nonequilibrium (quenched } \\
\text { from liquid) }\end{array}$ \\
\hline & $340{ }^{\circ} \mathrm{C}, 28$ days & $\begin{aligned} \eta & =\mathrm{Cu}_{2} \mathrm{Sb} \\
\theta & =(\mathrm{Sb})\end{aligned}$ & $\begin{array}{l}P 4 / n m m \\
R-3 m\end{array}$ & $\begin{array}{l}a=4.00172(3), c=6.10443(9) \\
a=4.3066(1), c=11.2689(6)\end{array}$ & \\
\hline & $470{ }^{\circ} \mathrm{C}, 28$ days & $\begin{array}{l}\eta=\mathrm{Cu}_{2} \mathrm{Sb} \\
\theta=(\mathrm{Sb})\end{array}$ & $\begin{array}{l}P 4 / n m m \\
R-3 m\end{array}$ & $\begin{array}{l}a=4.00204(2), c=6.10431(7) \\
a=4.30686(9), c=11.2708(5)\end{array}$ & \\
\hline & $600{ }^{\circ} \mathrm{C}, 28$ days & $\begin{aligned} \eta & =\mathrm{Cu}_{2} \mathrm{Sb} \\
\theta & =(\mathrm{Sb})\end{aligned}$ & $\begin{array}{l}P 4 / n m m \\
R-3 m\end{array}$ & $\begin{array}{l}a=4.00239(2), c=6.10462(8) \\
a=4.3072(1), c=11.2720(5)\end{array}$ & $\begin{array}{l}\text { Nonequilibrium (quenched } \\
\text { from liquid) }\end{array}$ \\
\hline \multirow[t]{2}{*}{$\mathrm{Cu}_{35} \mathrm{Sb}_{65}$} & $340{ }^{\circ} \mathrm{C}, 28$ days & $\begin{array}{l}\eta=\mathrm{Cu}_{2} \mathrm{Sb} \\
\theta=(\mathrm{Sb})\end{array}$ & $\begin{array}{l}P 4 / n m m \\
R-3 m\end{array}$ & $\begin{array}{l}a=4.00106(5), c=6.1026(1) \\
a=4.30569(6), c=11.2658(3)\end{array}$ & \\
\hline & $535^{\circ} \mathrm{C}, 28$ days & $\begin{array}{l}\eta=\mathrm{Cu}_{2} \mathrm{Sb} \\
\theta=(\mathrm{Sb})\end{array}$ & $\begin{array}{l}P 4 / n m m \\
R-3 m\end{array}$ & $\begin{array}{l}a=4.00165(5), c=6.1029(1) \\
a=4.30639(4), c=11.2707(2)\end{array}$ & $\begin{array}{l}\text { Nonequilibrium (quenched } \\
\text { from liquid) }\end{array}$ \\
\hline \multirow[t]{2}{*}{$\mathrm{Cu}_{30} \mathrm{Sb}_{70}$} & $340{ }^{\circ} \mathrm{C}, 28$ days & $\begin{array}{l}\eta=\mathrm{Cu}_{2} \mathrm{Sb} \\
\theta=(\mathrm{Sb})\end{array}$ & $\begin{array}{l}P 4 / n m m \\
R-3 m\end{array}$ & $\begin{array}{l}a=4.00218(4), c=6.1043(1) \\
a=4.30709(5), c=11.2701(2)\end{array}$ & \\
\hline & $535^{\circ} \mathrm{C}, 28$ days & $\begin{array}{l}\eta=\mathrm{Cu}_{2} \mathrm{Sb} \\
\theta=(\mathrm{Sb})\end{array}$ & $\begin{array}{l}P 4 / n m m \\
R-3 m\end{array}$ & $\begin{array}{l}a=4.00065(7), c=6.1018(2) \\
a=4.30563(4), c=11.2650(2)\end{array}$ & $\begin{array}{l}\text { Nonequilibrium (quenched } \\
\text { from liquid) }\end{array}$ \\
\hline \multirow[t]{2}{*}{$\mathrm{Cu}_{25} \mathrm{Sb}_{75}$} & $340{ }^{\circ} \mathrm{C}, 28$ days & $\begin{array}{l}\eta=\mathrm{Cu}_{2} \mathrm{Sb} \\
\theta=(\mathrm{Sb})\end{array}$ & $\begin{array}{l}P 4 / n m m \\
R-3 m\end{array}$ & $\begin{array}{l}a=4.00206(5), c=6.1040(1) \\
a=4.30703(4), c=11.2702(2)\end{array}$ & \\
\hline & $535^{\circ} \mathrm{C}, 28$ days & $\begin{aligned} \eta & =\mathrm{Cu}_{2} \mathrm{Sb} \\
\theta & =(\mathrm{Sb})\end{aligned}$ & $\begin{array}{l}P 4 / n m m \\
R-3 m\end{array}$ & $\begin{array}{l}a=4.00151(7), c=6.1029(2) \\
a=4.30667(7), c=11.2690(2)\end{array}$ & $\begin{array}{l}\text { Nonequilibrium (quenched } \\
\text { from liquid) }\end{array}$ \\
\hline
\end{tabular}

To investigate the solubility ranges of the phases, we performed SEM/EDX measurements on polished samples. We were especially interested in determining the ranges of homogeneity of the phases that had been only tentatively fixed in the literature ([3], dashed lines). All of the results of the EDX measurements along with BSE images of the examined samples can be found in Table 7. Overall, the ranges of homogeneity were found to fit well to the currently accepted phase diagram in the literature [3]. The solubility limits indicated by the dashed lines for the $\varepsilon$ phase, $\eta$ phase, and the high-temperature region of the $\delta$ phase were determined. For the $\varepsilon$ phase, an extension of the phase field to higher $\mathrm{Sb}$ concentrations than those estimated in the literature [3] was observed, and the $\eta$ phase was also found to occur at higher Sb concentrations (see Tables 7, 8; Fig. 4). Even the very narrow two-phase field between the $\delta$ and the $\zeta$ phases was confirmed by EDX and XRD measurements of the sample with 20.5 at\% Sb (see Table 5). 
Table 6 Detected phases in quenched samples

\begin{tabular}{|c|c|c|c|c|c|c|c|c|c|c|c|c|c|c|c|c|}
\hline \multirow[t]{2}{*}{ Sample } & \multicolumn{16}{|c|}{ Annealing temperature $\left({ }^{\circ} \mathrm{C}\right)$} \\
\hline & Melt & 170 & 280 & 340 & 350 & 420 & 390 & 400 & 430 & 420 & 435 & 450 & 470 & 480 & 535 & 600 \\
\hline $\mathrm{Cu}_{90} \mathrm{Sb}_{10}$ & $\underline{(\mathbf{C u}) \delta}$ & & & $(\mathrm{Cu}) \delta$ & & & $(\mathrm{Cu}) \delta$ & & & $(\mathrm{Cu}) \delta$ & $(\mathrm{Cu}) \delta$ & $(\mathrm{Cu}) \delta$ & $(C u) \beta, \gamma$ & (Cu) $\beta, \gamma$ & & $(C u) \gamma, \delta$ \\
\hline $\mathrm{Cu}_{82.5} \mathrm{Sb}_{17.5}$ & $\underline{\delta, \varepsilon}$ & & & $(\mathrm{Cu}) \delta$ & & & & & $\mathrm{Cu}, \delta$ & & & & $\gamma, \delta$ & & & $\underline{(\mathrm{Cu}) \beta, \delta}$ \\
\hline $\mathrm{Cu}_{80.5} \mathrm{Sb}_{19.5}$ & & & & $\delta$ & & & & & & & & & & & & \\
\hline $\mathrm{Cu}_{79.5} \mathrm{Sb}_{20.5}$ & & $\delta, \zeta, \eta$ & $\delta, \zeta$ & & $\delta, \zeta$ & $\delta, \varepsilon$ & & & & & & & & & & \\
\hline $\mathrm{Cu}_{79} \mathrm{Sb}_{21}$ & $\underline{\delta, \varepsilon}$ & & & $\zeta$ & & & & & $\delta, \varepsilon$ & & & & $\underline{\beta, \delta, \varepsilon}$ & & & \\
\hline $\mathrm{Cu}_{77.5} \mathrm{Sb}_{22.5}$ & $\underline{\delta, \varepsilon}$ & & & $\zeta, \eta$ & & & & & $\delta, \varepsilon$ & & & $\underline{\delta, \varepsilon, \eta}$ & $\underline{\delta, \varepsilon, \eta}$ & & & $\underline{\delta, \varepsilon, \eta}$ \\
\hline $\mathrm{Cu}_{76} \mathrm{Sb}_{24}$ & & $\delta, \eta$ & $\zeta, \eta$ & $\zeta, \eta$ & & & & $\varepsilon$ & & & & & & & & \\
\hline $\mathrm{Cu}_{74} \mathrm{Sb}_{26}$ & & $(\mathrm{Cu}) \delta, \zeta, \eta$ & $\zeta, \eta$ & $\zeta, \eta$ & & & & $\varepsilon, \eta$ & & & & & & & & \\
\hline $\mathrm{Cu}_{72} \mathrm{Sb}_{28}$ & $\underline{\varepsilon, \eta}$ & & & $\zeta, \eta$ & & & & & $\varepsilon, \eta$ & & & & & & & $\underline{\beta, \varepsilon, \eta}$ \\
\hline $\mathrm{Cu}_{70} \mathrm{Sb}_{30}$ & & & & & & & & & $\varepsilon, \eta$ & & & & $\underline{\beta, \varepsilon, \eta}$ & & & $\underline{\beta, \varepsilon, \eta}$ \\
\hline $\mathrm{Cu}_{60} \mathrm{Sb}_{40}$ & $\underline{\eta,(\mathrm{Sb})}$ & & & $\eta,(\mathrm{Sb})$ & & & & & & & & & $\eta,(\mathrm{Sb})$ & & & $\underline{\eta,(S b)}$ \\
\hline $\mathrm{Cu}_{35} \mathrm{Sb}_{65}$ & & & & $\eta,(\mathrm{Sb})$ & & & & & & & & & & & $\underline{\eta,(\mathrm{Sb})}$ & \\
\hline $\mathrm{Cu}_{30} \mathrm{Sb}_{70}$ & & & & $\eta,(\mathrm{Sb})$ & & & & & & & & & & & $\underline{\eta,(\mathrm{Sb})}$ & \\
\hline $\mathrm{Cu}_{25} \mathrm{Sb}_{75}$ & & & & $\eta,(\mathrm{Sb})$ & & & & & & & & & & & $\underline{\eta,(\mathrm{Sb})}$ & \\
\hline
\end{tabular}

Bold-underlined quenched from liquid, bold insufficiently annealed, italic-underlined decomposition of $\beta$ phase
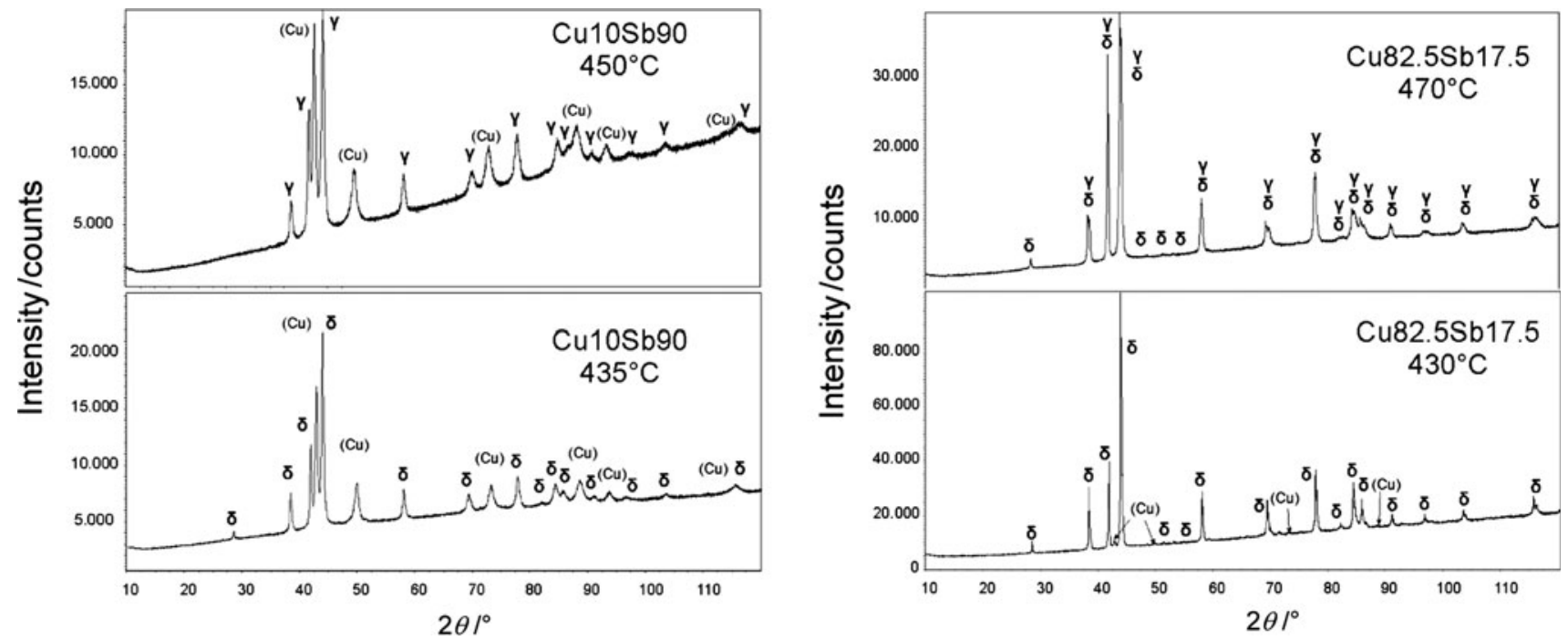

Fig. 3 XRD patterns of quenched $\mathrm{Cu}_{90} \mathrm{Sb}_{10}$ and $\mathrm{Cu}_{82.5} \mathrm{Sb}_{17.5}$ samples annealed at different temperatures

\section{Experimental}

\section{Sample preparation}

Samples with 10-75 at $\%$ Sb (see Table 9) were prepared from $99.98 \% \mathrm{Cu}$ (Goodfellow, Cambridge, UK; treated under an $\mathrm{H}_{2}$ flow at $200{ }^{\circ} \mathrm{C}$ for $5 \mathrm{~h}$ to remove oxide layers) and $99.999 \% \mathrm{Sb}$ (Alfa Aesar, Karlsruhe, Germany; the surface oxide layer was removed by filtration of the melt through quartz glass wool). Weighed amounts of the metals were sealed in quartz glass ampoules under vacuum $\left(\sim 10^{-3}\right.$ mbar $)$ and alloyed in a resistance furnace at $1,000{ }^{\circ} \mathrm{C}$ for a few hours. Annealing was performed again in evacuated quartz glass ampoules for 28 days at selected temperatures $\left(170-600{ }^{\circ} \mathrm{C}\right.$, annealing time at $170{ }^{\circ} \mathrm{C}$ was 6 months). Finally, the alloys were quenched in cold water.

\section{Analytical methods}

Experimental techniques applied were powder X-ray diffraction (XRD), thermal analysis (DTA), and metallographic methods (EPMA/ESEM). Thermal analysis was 
done with a TG/DTA Setsys Evolution instrument from Setaram. The measurements were performed in open alumina crucibles under an $\mathrm{Ar}$ atmosphere; slices of $\mathrm{Ti}$ sheet in the second crucible were used as reference material.
The powder XRD measurements were done on a Bruker D8 diffractometer ( $\theta / 2 \theta$ geometry) at ambient temperature. $\mathrm{X}$-rays were produced in a copper radiation source at an accelerating voltage of $40 \mathrm{kV}$ and with an electron current of $40 \mathrm{~mA}$. A Ni filter was used to remove the $\mathrm{K}_{\beta}$ radiation.

Table 7 ESEM/EPMA results of $\mathrm{Cu}-\mathrm{Sb}$ phase compositions

\begin{tabular}{|c|c|c|c|c|c|c|c|c|}
\hline \multirow[t]{2}{*}{ Sample } & \multirow[t]{2}{*}{ Ann. temp. $/{ }^{\circ} \mathrm{C}$} & \multicolumn{3}{|c|}{ Phase 1 (dark) } & \multicolumn{3}{|c|}{ Phase 2 (bright) } & \multirow[t]{2}{*}{ SEM imag } \\
\hline & & & at $\% \mathrm{Cu}$ & at $\% \mathrm{Sb}$ & & at $\% \mathrm{Cu}$ & at $\% \mathrm{Sb}$ & \\
\hline $\mathrm{Cu}_{90} \mathrm{Sb}_{10}$ & 600 & $(\mathrm{Cu})$ & 93.0 & 7.0 & $\beta_{\mathrm{Cu}}$ & 80.2 & 19.8 & \\
\hline
\end{tabular}

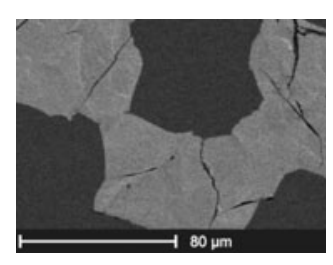

17.7

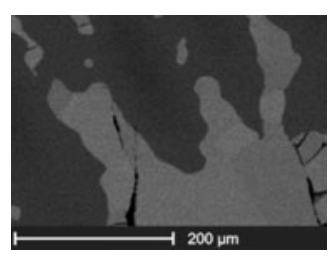

21.2
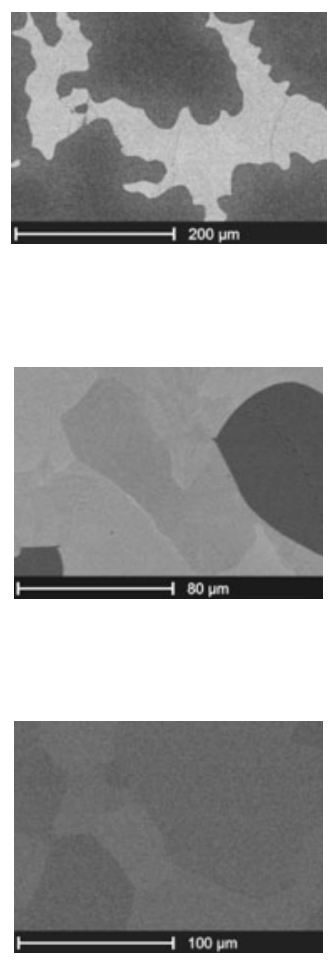
Table 7 continued

\begin{tabular}{|c|c|c|c|c|c|c|c|c|}
\hline \multirow[t]{2}{*}{ Sample } & \multirow[t]{2}{*}{ Ann. temp. $/{ }^{\circ} \mathrm{C}$} & \multicolumn{3}{|l|}{ Phase 1 (dark) } & \multicolumn{3}{|c|}{ Phase 2 (bright) } & \multirow[t]{2}{*}{ SEM image } \\
\hline & & & at $\% \mathrm{Cu}$ & at $\% \mathrm{Sb}$ & & at $\% \mathrm{Cu}$ & at $\% \mathrm{Sb}$ & \\
\hline $\mathrm{Cu}_{79.5} \mathrm{Sb}_{20.5}$ & 350 & $\zeta_{\mathrm{Cu}}$ & 77.2 & 22.8 & $\delta_{\mathrm{Sb}}$ & 78.0 & 22.0 & \\
\hline
\end{tabular}

$\mathrm{Cu}_{79} \mathrm{Sb}_{21} \quad 470 \quad \delta_{\mathrm{Sb}}$

$\mathrm{Cu}_{77.5} \mathrm{Sb}_{22.5} \quad 430$

$\delta_{\mathrm{Sb}}$

79.6

20.4

$\varepsilon_{\mathrm{Cu}}$

76.7

23.4

$\mathrm{Cu}_{76} \mathrm{Sb}_{24} \quad 170$

$\delta_{\mathrm{Sb}}(+$ small crystals of $\eta)$

77.5

22.5

$\eta_{\mathrm{Cu}}$

66

34.0

$\mathrm{Cu}_{74} \mathrm{Sb}_{26} \quad 400$

$\varepsilon_{\mathrm{Sb}}$

74.4

25.6

$\eta_{\mathrm{Cu}}$

65.2

34.8
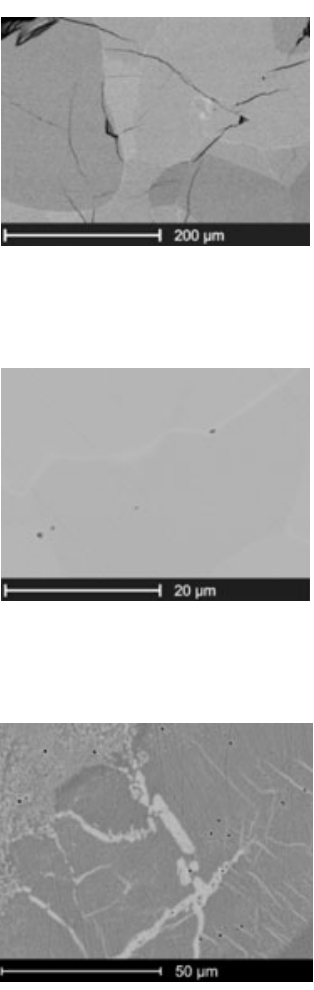

$50 \mathrm{~mm}$

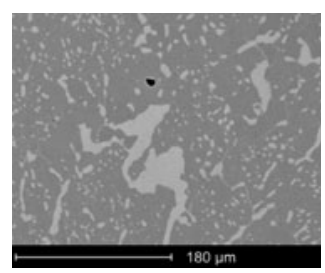


Table 7 continued

\begin{tabular}{|c|c|c|c|c|c|c|c|c|}
\hline \multirow[t]{2}{*}{ Sample } & \multirow[t]{2}{*}{ Ann. temp. $/{ }^{\circ} \mathrm{C}$} & \multicolumn{3}{|l|}{ Phase 1 (dark) } & \multicolumn{3}{|c|}{ Phase 2 (bright) } & \multirow[t]{2}{*}{ SEM image } \\
\hline & & & at $\% \mathrm{Cu}$ & at $\% \mathrm{Sb}$ & & at $\% \mathrm{Cu}$ & at $\% \mathrm{Sb}$ & \\
\hline $\mathrm{Cu}_{72} \mathrm{Sb}_{28}$ & 430 & $\varepsilon_{\mathrm{Sb}}$ & 73.7 & 26.3 & $\eta_{\mathrm{Cu}}$ & 64.7 & 35.3 & \\
\hline
\end{tabular}

$\begin{array}{lllllll}340 & \zeta_{\mathrm{Sb}} & 78.0 & 22.0 & \eta_{\mathrm{Cu}} & 65.5 & 34.5\end{array}$

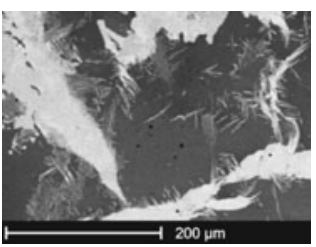

$\begin{array}{llllllll}\mathrm{Cu}_{60} \mathrm{Sb}_{40} & 470 & \eta_{\mathrm{Sb}} & 64 & 36 & (\mathrm{Sb}) & 0.1 & 99.9\end{array}$

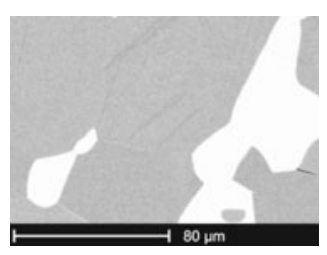

Table 8 Comparison of temperature-invariant reactions in the $\mathrm{Cu}-\mathrm{Sb}$ system in this work and in [1]

\begin{tabular}{|c|c|c|c|c|c|}
\hline \multirow{2}{*}{$\frac{\text { Reaction }}{\mathrm{L} \rightarrow \beta}$} & \multicolumn{3}{|c|}{ Composition /at\% Sb } & \multirow{2}{*}{$\frac{\text { Temp. } /{ }^{\circ} \mathrm{C}}{690(683)}$} & \multirow{2}{*}{$\frac{\text { Reaction type }}{\text { Congruent melt }}$} \\
\hline & & $29.0(29.0)$ & & & \\
\hline $\mathrm{L} \rightarrow(\mathrm{Cu})+\beta$ & $7.8(5.8)$ & 17.7 (19) & $20.4(19.5)$ & $645(645)$ & Eutectic \\
\hline $\mathrm{L}+\beta \rightarrow \eta$ & $31.0^{\mathrm{b}}$ & $35.5(33.5)$ & $46.0^{\mathrm{b}}$ & $586(586)$ & Peritectic \\
\hline $\mathrm{L} \rightarrow \eta+(\mathrm{Sb})$ & $35.5(33.5)$ & $63.0^{\mathrm{b}}$ & $99.9(99.9)$ & $526(526)$ & Eutectic \\
\hline$(\mathrm{Cu})+\beta \rightarrow \gamma$ & $6.4(5.65)$ & $16.5(15.5)$ & $20.7(21.5)$ & $484(488)$ & Peritectoid \\
\hline$\beta+\gamma \rightarrow \delta$ & $17.5(16.5)$ & $19.5(19.0)$ & $21.5(24.0)$ & $467(462)$ & Peritectoid \\
\hline$\beta+\delta \rightarrow \varepsilon$ & $20.2(19.5)$ & $22.3(23.0)$ & $23.9(25.5)$ & $440\left(445^{\mathrm{a}}\right)$ & Peritectoid \\
\hline$\beta \rightarrow \varepsilon+\eta$ & $26.3(25.5)$ & $29.3(26.5)$ & $34.2(32.0)$ & $427\left(440^{\mathrm{a}}\right)$ & Eutectoid \\
\hline$\gamma \rightarrow(\mathrm{Cu})+\delta$ & $5.0(4.6)$ & $16.5(15.5)$ & $19.0(18.5)$ & $440(400)$ & Eutectoid \\
\hline$\delta+\varepsilon \rightarrow \zeta$ & $20.2(20.0)$ & $20.8(21.5)$ & $23.2(23.5)$ & $390^{\mathrm{a}, \mathrm{b}}$ & Peritectoid \\
\hline$\varepsilon \rightarrow \zeta+\eta$ & $22.2(22.5)$ & $24.0(24.0)$ & $34.4(32.5)$ & $360(360)$ & Eutectoid \\
\hline$\zeta \rightarrow \delta+\eta$ & $20.2(20.0)$ & $20.8(21.5)$ & $34.4(32.5)$ & $260\left(260^{\mathrm{a}}\right)$ & Eutectoid \\
\hline
\end{tabular}

Values in parentheses are from the literature [1]

a Uncertain values

b Value from [1] 


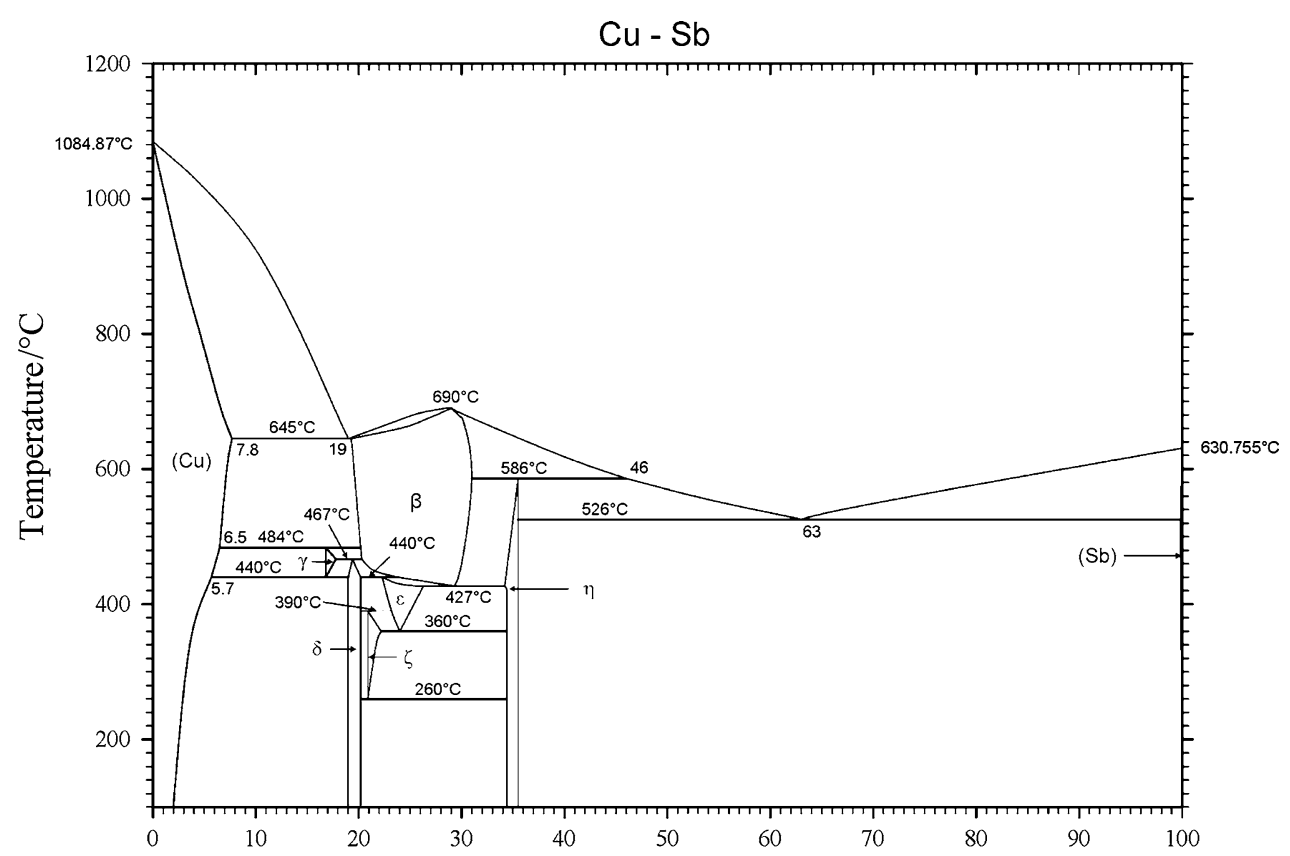

$\mathrm{Cu}$

Atomic percent antimony

$\mathrm{Sb}$

Fig. 4 New version of the $\mathrm{Cu}-\mathrm{Sb}$ phase diagram

Table 9 Annealing temperatures

\begin{tabular}{|c|c|c|c|c|c|c|c|c|c|c|c|c|c|c|c|}
\hline \multirow{2}{*}{$\frac{\text { Sample }}{\mathrm{Cu}_{90} \mathrm{Sb}_{10}}$} & \multicolumn{15}{|c|}{ Annealing temperature $/{ }^{\circ} \mathrm{C}$} \\
\hline & Melt & & & 340 & & & 390 & & 420 & & $\underline{435}$ & 450 & 470 & 480 & 600 \\
\hline $\mathrm{Cu}_{82.5} \mathrm{Sb}_{17.5}$ & Melt & & & 340 & & & & & & 430 & & & 470 & & 600 \\
\hline $\mathrm{Cu}_{80.5} \mathrm{Sb}_{19.5}$ & Melt & & & 340 & & & & & & & & & & & \\
\hline $\mathrm{Cu}_{79.5} \mathrm{Sb}_{20.5}$ & Melt & 170 & 280 & & 350 & 420 & & & & & & & & & \\
\hline $\mathrm{Cu}_{79} \mathrm{Sb}_{21}$ & Melt & & & 340 & & & & & & 430 & & & 470 & & \\
\hline $\mathrm{Cu}_{77.5} \mathrm{Sb}_{22.5}$ & Melt & & & 340 & & & & & & 430 & & 450 & 470 & & 600 \\
\hline $\mathrm{Cu}_{76} \mathrm{Sb}_{24}$ & Melt & 170 & 280 & 340 & & & & 400 & & & & & & & \\
\hline $\mathrm{Cu}_{74} \mathrm{Sb}_{26}$ & Melt & 170 & 280 & 340 & & & & 400 & & & & & & & \\
\hline $\mathrm{Cu}_{72} \mathrm{Sb}_{28}$ & Melt & & & 340 & & & & & & 430 & & & & & 600 \\
\hline $\mathrm{Cu}_{70} \mathrm{Sb}_{30}$ & Melt & & & & & & & & & 430 & & & 470 & & 600 \\
\hline $\mathrm{Cu}_{60} \mathrm{Sb}_{40}$ & Melt & & & 340 & & & & & & & & & 470 & & 600 \\
\hline $\mathrm{Cu}_{35} \mathrm{Sb}_{65}$ & Melt & & & 340 & & & & & & & & & & & \\
\hline $\mathrm{Cu}_{30} \mathrm{Sb}_{70}$ & Melt & & & 340 & & & & & & & & & & & \\
\hline $\mathrm{Cu}_{25} \mathrm{Sb}_{75}$ & Melt & & & 340 & & & & & & & & & & & \\
\hline
\end{tabular}

Underlined only XRD, bold XRD/ESEM, italics XRD/ESEM/DTA

The powder was fixed with petroleum jelly on a silicon monocrystal sample carrier, which was rotated during the measurement. The detection unit was the Lynxeye strip detector. Rietveld refinement of the data was done with the Topas $3{ }^{\circledR}$ software provided by Bruker AXS.

An optical microscope (Zeiss Axiotech 100 reflected light microscope) as well as EDX techniques (energy- dispersive spectroscopy; ESEM Zeiss Supra 55 VP) were used for metallographic investigations. In the ESEM, the excitation energy of the electron beam was $15-20 \mathrm{kV}$. Backscattered electrons were detected in order to visualize the surfaces of our samples. The characteristic spectral lines were used for EDX: the $\mathrm{Cu} \mathrm{K}$ line and the $\mathrm{Sb} \mathrm{L}$ line. 
Acknowledgments We wish to thank the FWF (Fonds zur Förderung der wissenschaftlichen Forschung), who provided the funds for this work through the project P21507-N19. Many thanks also to Dr. Stephan Puchegger from the Center for Nano Structure Research, University of Vienna, for supporting our SEM/EDX measurements.

Open Access This article is distributed under the terms of the Creative Commons Attribution License which permits any use, distribution, and reproduction in any medium, provided the original author(s) and the source are credited.

\section{References}

1. Gierlotka W, Jendrzejczyk-Handzlik D (2009) J Alloys Compd 484:172

2. Liu XJ, Wang CP, Ohnuma I, Kainuma R, Ishida K (2000) J Phase Equilib 21:432

3. Massalski TB (1990) $\mathrm{Cu}-\mathrm{Sb}$ (copper-antimony). In: Binary alloy phase diagrams, vol 2, 2nd edn. The Materials Information Society, Materials Park

4. Suh IK, Ohta H, Waseda Y (1988) High temperature thermal expansion of six metallic elements measured by dilatation method and X-ray diffraction. In: Villars P, Calvert LD, Pearson WB (eds) Pearson's handbook of crystallographic data for intermetallic phases, vol 3, 2nd edn. The Materials Information Society, Materials Park

5. Hofmann W (1941) Zur Überstruktur von $\mathrm{Cu}_{3} \mathrm{Sb}$. In: Villars $\mathrm{P}$, Calvert LD, Pearson WB (eds) Pearson's handbook of crystallographic data for intermetallic phases, vol 3, 2nd edn. The Materials Information Society, Materials Park
6. Schubert K, Breimer H, Burkhardt W, Günzel E, Haufler R, Lukas HL, Vetter H, Wegst J, Wilkens M (1957) Einige strukturelle ergebnisse an metallischen phasen II. In: Villars P, Calvert LD, Pearson WB (eds) Pearson's handbook of crystallographic data for intermetallic phases, vol 3, 2nd edn. The Materials Information Society, Materials Park

7. Yamaguchi S, Hirabayashi M (1972) J Phys Soc Jpn 33:708

8. Karlsson N (1972) Acta Crystallogr B 28:371

9. Günzel E, Schubert K (1958) Strukturuntersuchungen im system kupfer-antimon. In: Villars P, Calvert LD, Pearson WB (eds) Pearson's handbook of crystallographic data for intermetallic phases, vol 3, 2nd edn. The Materials Information Society, Materials Park

10. Pearson WB (1964) Electrical resistivity, Hall coefficient, and thermoelectric power of $\mathrm{AuSb}_{2}$ and $\mathrm{Cu}_{2} \mathrm{Sb}$. In: Villars $\mathrm{P}$, Calvert LD, Pearson WB (eds) Pearson's handbook of crystallographic data for intermetallic phases, vol 3, 2nd edn. The Materials Information Society, Materials Park

11. Barrett CS, Cucka P, Haefner K (1963) The crystal structure of antimony at $4.2,78$, and $298 \mathrm{~K}$. In: Villars P, Calvert LD, Pearson WB (eds) Pearson's handbook of crystallographic data for intermetallic phases, vol 4, 2nd edn. The Materials Information Society, Materials Park

12. Schubert K, Ilschner M (1954) Z Metallkd 45:366

13. Heumann T, Heinemann F (1956) Z Elektrochem 60:1160

14. Hansen M (ed) (1958) Constitution of binary alloys, 2nd edn. McGraw-Hill, New York

15. Günzel E, Schubert K (1958) Z Metallkd 49:124

16. Murakami T, Shibata N (1936) Science reports of the Tohoku University, series 1, vol 25. Tohoku University, Sendai, p 527 